\title{
The Impact of Primary Tumor Resection on Survival in Asymptomatic Colorectal Cancer Patients With Unresectable Metastases
}

\author{
Ki Yoon Doah, Ui Sup Shin, Byong Ho Jeon, Sang Sik Cho, Sun Mi Moon \\ Department of Surgery, Korea Institute of Radiological \& Medical Sciences, Korea Cancer Center Hospital, Seoul, Korea
}

Purpose: This study was conducted to evaluate the effectiveness of primary tumor resection (PTR) in asymptomatic colorectal cancer $(\mathrm{CRC})$ patients with unresectable metastases using the inverse probability of treatment weighting (IPTW) method to minimize selection bias.

Methods: We selected 146 patients diagnosed with stage IV CRC with unresectable metastasis between 2001 and 2018 from our institutional database. In a multivariate logistic regression model using the patients' baseline covariates associated with PTR, we applied the IPTW method based on a propensity score and performed a weighted Cox proportional regression analysis to estimate survival according to PTR.

Results: Upfront PTR was performed in 98 patients, and no significant differences in baseline factors were detected. The upweighted median survival of the PTR group was 18 months and that of the non-PTR group was 15 months $(\mathrm{P}=0.15)$. After applying the IPTW, the PTR was still insignificant in the univariate Cox regression (hazard ratio [HR], 0.26; 95\% confidence interval $[\mathrm{CI}], 0.5-1.21)$. However, in the multivariate weighted Cox regression with adjustment for other covariates, the PTR showed a significantly decreased risk of cancer-related death (HR, 0.61; 95\% CI, 0.40-0.94).

Conclusion: In this study, we showed that asymptomatic CRC patients with unresectable metastases could gain a survival benefit from upfront PTR by analysis with the IPTW method. However, randomized controlled trials are mandatory.

\section{Keywords: Colonic neoplasms; Surgery; Neoplasm metastasis; Prognosis; Probability; Selection bias}

\section{INTRODUCTION}

According to reports of the 2018 International Agency for Research on Cancer, colorectal cancer (CRC) is the third most common cancer worldwide. In this report, the Korean incidence of $\mathrm{CRC}$ was the highest worldwide, although there has been a recent decrease in the annual domestic statistics $[1,2]$. When the disease is diagnosed without metastasis, the 5-year survival rate is over

Received: Jul 22, 2020 - Revised: Sep 15, 2020 • Accepted: Sep 15, 2020 Correspondence to: Ui Sup Shin, M.D.

Department of Surgery, Korea Institute of Radiological \& Medical Sciences, Korea Cancer Center Hospital, 75 Nowon-ro, Nowon-gu, Seoul 01812, Korea Tel: +82-2-970-1216, Fax: +82-2-970-2419

E-mail: uisupshin@kirams.re.kr

ORCID: https://orcid.org/0000-0002-1714-7469

(C) 2021 The Korean Society of Coloproctology

This is an open-access article distributed under the terms of the Creative Commons Attribution NonCommercial License (https://creativecommons.org/licenses/by-nc/4.0) which permits unrestricted noncommercial use, distribution, and reproduction in any medium, provided the original work is properly cited.
$70 \%$. However, more than $20 \%$ of CRC cases are found with distant metastases, and overall survival (OS) is only about $14 \%$ to $20 \%$ [3]. Even for stage IV CRC, radical resection, including metastatic lesions, is mandatory to achieve long-term survival. However, in $65 \%$ of patients with stage IV CRC, radical surgery is not possible [4]. Upfront active chemotherapy, including various biological agents, is recommended in the current treatment guidelines [5]. However, primary tumor resection (PTR) or other palliative surgical treatments such as stoma formation or bypass surgeries are inevitable in cases of complications such as intestinal obstruction, hemorrhage, or perforation for symptomatic relief and further medical treatment. In asymptomatic patients at the time of diagnosis, many clinicians have performed upfront PTR before chemotherapy [6] because of the concern of future complications from the primary lesion during chemotherapy despite the debate about the usefulness of PTR in those situations [4, 6-15].

The treatment for these cases should be provided by a multidisciplinary team approach consisting of surgical, medical, and radi- 
ation oncologists, along with pathologists and radiologists [16, 17]. By considering the resectability, location, and size of the primary/metastatic tumor, the treatment strategies are decided in consideration with the patient's preference, socioeconomic status, symptoms, and general condition. Therefore, various combinations of medical or nonmedical pretreatment factors can be involved in treatment decisions. To date, the reason why the debate about the advantage of PTR for asymptomatic CRC patients with unresectable metastasis continues is because of the lack of confirmative results of a well-controlled randomized trial $[18,19]$. For studies supporting PTR, most points of criticism have been about their selection bias because of the various pretreatment factors mentioned earlier, which cannot be easily controlled [20].

In this study, we evaluated the effectiveness of PTR using the IPTW method, a recently emerging statistical tool to minimize selection bias by adjusting the baseline confounders.

\section{METHODS}

\section{Patients and treatments}

We selected 245 patients who were diagnosed with stage IV CRC with unresectable metastasis between 2001 and 2018 from our institutional CRC database. The staging workups consisted of computed tomography (CT), including the chest, abdomen, and pelvis, and positron emission tomography fused with $\mathrm{CT}$ after tissue confirmation by endoscopic biopsy. At the clinician's discretion, pelvic or liver magnetic resonance imaging was performed if necessary. The resectability of the primary and the metastatic lesions was assessed by reviewing the radiological findings. Whether the patient was symptomatic or not was determined by reviewing the medical records and radiologic results. Symptoms of the primary lesion were defined as follows: obstruction was determined when gas distention existed at a bowel site proximal to the primary lesion; defecation difficulty was defined as a large amount of fecal impaction without gas distension; perforation/abscess was defined as free air in the abdominal cavity or abnormal fluid collection around the primary tumor with any systemic inflammatory sign or symptoms; bleeding was defined as hemorrhage from the primary lesion that required blood transfusion to maintain stable blood pressure. After reviewing the medical records and radiologic results, we excluded 91 symptomatic patients and 8 patients with unresectable primary lesions. Our Institutional Review Board approved this study, and the requirement for informed consent was waived because of the retrospective nature of the study (No. 2020-05-005).

Surgical treatment consisted of PTR, stoma formation, and bypass surgery. PTR was performed in the same manner as surgery, indicating nonmetastatic CRC with a possible level of lymphadenectomy. For the intention-to-treat analysis, the patients who underwent PTR during the course of chemotherapy were classified as the non-PTR group.

Chemotherapies were classified as monotherapy (fluorouracil or capecitabine only), doublet chemotherapy (monotherapy coupled with irinotecan or oxaliplatin), and doublet chemotherapy with a biologic agent (bevacizumab or cetuximab).

\section{Statistical analysis}

For the description of the study population, the chi-square or Fisher exact test was used to compare the categorical variables. For continuous variables, independent or paired t-test and the Wilcoxon rank-sum or signed-rank tests were used appropriately as required.

To identify the possible factors that influence the decision of performing PTR or non-PTR, we performed a multivariate binary logistic regression and calculated the propensity score (PS). Then, the inverse probability treatment weighting (IPTW) method was applied to minimize the selection bias. The variables for estimating the PS were as follows; sex, age, pretreatment Charlson comorbidity score, level of carcinoembryonic antigen, primary tumor location, organs involved in metastasis, multiple organ metastases, and chemotherapy. To check the goodness of fit and the discriminability of the model employed for calculating the PS, we used the Hosmer and Lemeshow test and the receiveroperating curve (ROC) analysis. The weights designated to each patient were estimated using the following formula: for the PTR group, the weights were allocated as 1; and for the non-PTR group, the weights were calculated as PS/(1 - PS) [21].

To check the imbalance of covariates between the PTR and nonPTR groups, the standardized mean difference (SMD) was calculated before and after weighting, and an absolute value of SMD less than 0.2 was considered balanced [22].

As the endpoint of this study, the OS was defined as the time from the date of diagnosis of stage IV CRC to the date of cancerrelated death. For survival analysis and comparison, the KaplanMeier and log-rank tests were used. Cox proportional hazard regression analysis with/without weighting was performed for the univariate and multivariate analyses. Variables with a P-value of $<0.2$ in the weighted univariate analysis were selected for weighted multivariate analysis. For the multivariate analysis, a backward stepwise elimination method was used. All tests were 2 -sided, and a P-value of $<0.05$ was considered significant. $\mathrm{R}$ software ver. 4.0.0 (R Foundation for Statistical Computing, Vienna, Austria) was used for the statistical analyses.

\section{RESULTS}

\section{Patients' characteristics}

The median age of the 146 patients was 68.5 years (interquartile range [IQR], 59-76 years). The number of male patients was 78 (53.4\%). The median Charlson comorbidity index (CCI) score was 3.0 (IQR, 2.0-4.0). The most common metastatic organ was the liver (70.5\%), and peritoneal metastasis was observed in 29 patients (19.9\%). Chemotherapy was administered to 121 patients (82.9\%). The number of patients who received doublet chemo- 
therapy or targeted therapy was 106 (72.6\%). Surgical treatment was performed in 140 patients (95.9\%). In addition, 110 patients (75.3\%) underwent PTR. Among them, 12 patients underwent upfront chemotherapy before PTR, and they were classified into the non-PTR group for intention-to-treat analysis. Twenty-seven patients with non-PTR $(\mathrm{n}=48)$ underwent upfront chemotherapy. Among them, 13 patients (48.1\%) received surgical treatment because of complications of the primary lesion (PTR, 4; stoma/bypass, 9). Among the 98 upfront PTR patients, 1 patient developed anastomotic leakage after low anterior resection, 2 patients underwent wound evisceration after surgery, and there was no opera- tion-related mortality. After upfront PTR, 80 patients (81.6\%) received chemotherapy. The median chemotherapy initiation time was 29 days (IQR, 21-36 days) after upfront PTR (Table 1).

\section{Balance between the PTR and non-PTR groups}

Although there were no significant differences in the baseline characteristics between the groups in the upweighted descriptive analysis (Table 1), there were potentially important imbalances in the 3 covariates with SMD $>0.2$ (Fig. 1); the levels of carcinoembryonic antigen $(\mathrm{SMD}=0.41)$, the presence of distant lymph node metastasis $(\mathrm{SMD}=0.36)$, and the primary tumor location in the

Table 1. Descriptive analyses between the primary tumor resection (PTR) vs. non-PTR group

\begin{tabular}{|c|c|c|c|c|}
\hline Variable & PTR & Non-PTR & Total & P-value \\
\hline No. of patients & 98 & 48 & 146 & \\
\hline Male sex & $49(50.0)$ & $29(60.4)$ & 78 (53.4) & 0.313 \\
\hline Age (yr) & $69.0(58.0-77.0)$ & $66.5(62.0-75.0)$ & $68.5(59.0-76.0)$ & 0.881 \\
\hline Charlson comorbidity index & $3.0(2.0-4.0)$ & $3.0(2.0-4.0)$ & $3.0(2.0-4.0)$ & 0.541 \\
\hline Level of CEA (ng/dL) & $24.2(4.7-123.5)$ & $16.9(4.8-105.5)$ & $20.4(4.7-120.2)$ & 0.452 \\
\hline Location of primary lesion & & & & 0.262 \\
\hline Right colon & 29 (29.6) & $10(20.8)$ & $39(26.7)$ & \\
\hline Left colon & 39 (39.8) & $17(35.4)$ & $56(38.4)$ & \\
\hline Rectum & $30(30.6)$ & $21(43.8)$ & $51(34.9)$ & \\
\hline Multiple organ metastases & $43(43.9)$ & $21(43.8)$ & $64(43.8)$ & 1.000 \\
\hline \multicolumn{5}{|l|}{ Site of metastasis } \\
\hline Liver & $72(73.5)$ & $31(64.6)$ & $103(70.5)$ & 0.361 \\
\hline Lung & $37(37.8)$ & $17(35.4)$ & $54(37.0)$ & 0.926 \\
\hline Peritoneum & 19 (19.4) & $10(20.8)$ & $29(19.9)$ & 1.000 \\
\hline Distant lymph node & $17(17.3)$ & $15(31.2)$ & $32(21.9)$ & 0.09 \\
\hline Bone & $6(6.1)$ & $1(2.1)$ & $7(4.8)$ & 0.509 \\
\hline Ovary & $7(7.1)$ & $1(2.1)$ & $8(5.5)$ & 0.382 \\
\hline Others & $2(2.0)$ & $1(2.1)$ & $3(2.1)$ & 1.000 \\
\hline Surgical treatment & & & & $<0.0001^{*}$ \\
\hline None & $0(0)$ & $6(12.5)$ & $6(4.1)$ & \\
\hline PTR & $98(100)$ & $12(25.0)$ & $110(75.3)$ & \\
\hline Stoma/bypass & $0(0)$ & $30(62.5)$ & $30(20.5)$ & \\
\hline Chemotherapy, yes & $80(81.6)$ & $41(85.4)$ & 121 (82.9) & 0.737 \\
\hline First-line regimen & & & & 0.644 \\
\hline None & $18(18.4)$ & $7(14.6)$ & $25(17.1)$ & \\
\hline Monotherapy & $9(9.2)$ & $6(12.5)$ & $15(10.3)$ & \\
\hline Doublet & $57(58.2)$ & $25(52.1)$ & $82(56.2)$ & \\
\hline Biologic agent & $14(14.3)$ & $10(20.8)$ & $24(16.4)$ & \\
\hline Upfront chemotherapy, yes & $0(0)$ & $27(56.2)$ & 27 (18.5) & $<0.0001^{*}$ \\
\hline
\end{tabular}

Values are presented as number only, number (\%), or median (interquartile range). CEA, carcinoembryonic antigen.

${ }^{*}$ Statistically significant $(P<0.05)$. 
Volume 37, Number 2, 2021

rectum $(S M D=0.32)$. As these could be a major source of bias for further comparison, we constructed a PS to predict probabilities of treatment allocation with baseline covariates by a multivariable logistic regression and calculated the weights for applying the

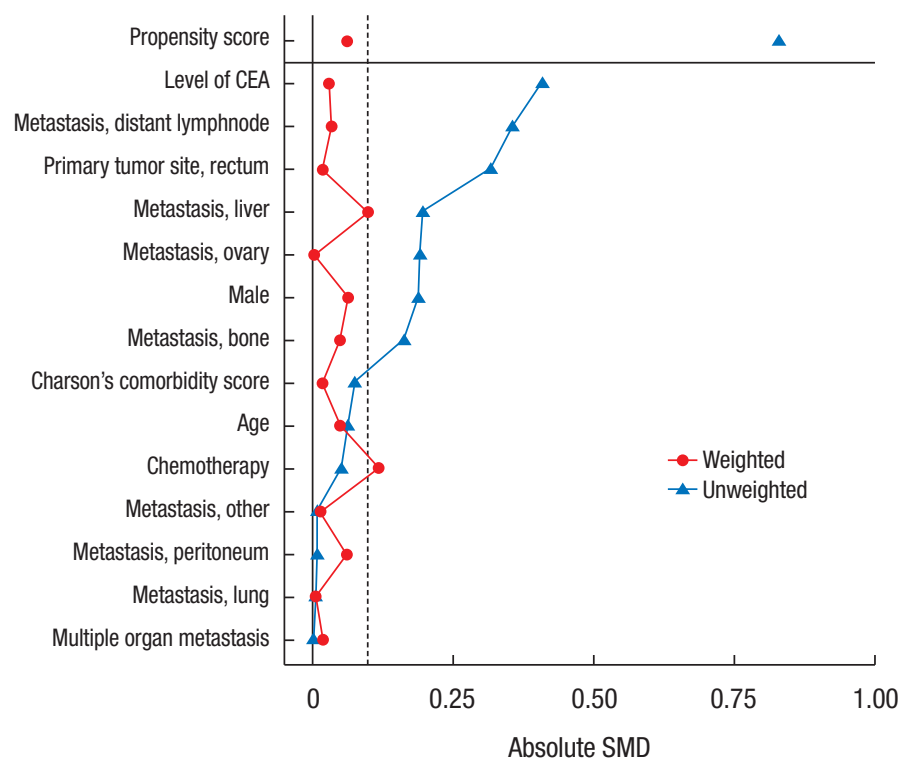

Fig. 1. Standardized mean difference (SMD) of baseline covariates before and after inverse probability treatment weighting. CEA, carcinoembryonic antigen.
IPTW as described in the method section. This treatment allocation model showed acceptable adequacy with a Hosmer and Lemeshow goodness of fit test $\left(\chi^{2}=6.7\right.$, degree of freedom $=8$, $\mathrm{P}=0.57$ ) and discrimination with an area under the ROC of 0.72 . After weighing all 14 covariates, SMDs were less than 0.2 and were considered to be well balanced (Fig. 1).

\section{Survival analysis}

During the median 18 months (IQR, 11-29 months), 132 of the 146 patients died because of disease, and the median survival time was 18 months. Comparing the survival according to whether the PTR was performed in the upweighted population, the median survival months of the PTR group was 18 months, and that of the non-PTR group was 15 months $(\mathrm{P}=0.152)$.

In the univariate Cox regression analysis without weights, the age (hazard ratio [HR], 1.03; 95\% confidence interval [CI], 1.011.05], CCI score (HR, 1.26; 95\% CI, 1.10-1.44), and chemotherapy (HR, 0.5 ; 95\% CI, 0.36-0.9) were found to be significant coprognostic factors affecting cancer-specific survival. The PTR showed a decreased risk of death but was not significant (HR, 0.77; 95\% CI, 0.53-1.13). In the weighted univariate Cox regression, the significant prognostic factors were age (HR, 1.03; $95 \%$ CI, 1.01-1.05), CCI score (HR, 1.25; 95\% CI, 1.04-1.5), the organ of metastasis in spleen, brain (indicated as others) (HR, 2.06; 95\% CI, 1.01-4.21), multiple organ metastasis (HR, 1.53; 95\% CI, 1.03-2.29), and chemotherapy (HR, 0.49; 95\% CI, 0.26-0.94). The PTR was still an insignificant factor in this univariate weighted

Table 2. Univariate Cox analysis of the factors associated with cancer-related survival

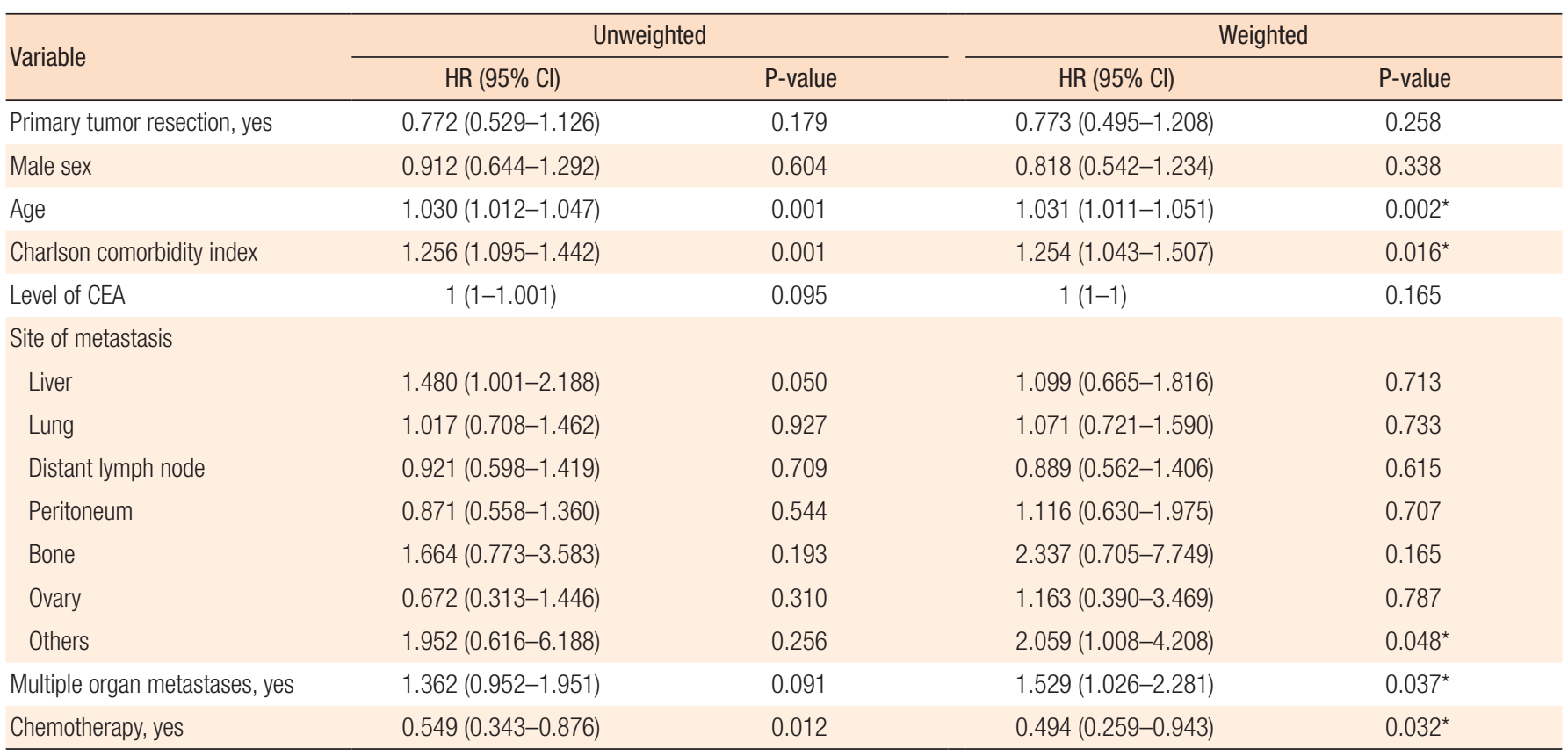

$\mathrm{HR}$, hazard ratio; $\mathrm{Cl}$, confidence interval; CEA, carcinoembryonic antigen.

*Statistically significant $(\mathrm{P}<0.05)$. 
Table 3. Multivariate Cox analysis of the factors associated with cancer-related survival

\begin{tabular}{lll}
\hline Variable & \multicolumn{1}{c}{ HR $(95 \% \mathrm{Cl})$} & P-value \\
\hline Primary tumor resection, yes & $0.614(0.402-0.938)$ & $0.024^{*}$ \\
Age & $1.030(1.007-1.054)$ & $0.012^{*}$ \\
Metastasis, bone & $2.592(0.84-7.992)$ & 0.097 \\
Multiple organ metastases, yes & $1.736(1.121-2.688)$ & $0.013^{*}$ \\
Chemotherapy, yes & $0.570(0.292-1.116)$ & 0.101 \\
\hline
\end{tabular}

$\mathrm{HR}$, hazard ratio; $\mathrm{Cl}$, confidence interval.

*Statistically significant $(P<0.05)$.

Cox regression (HR, 0.26; 95\% CI, 0.5-1.21) (Table 2).

However, in the multivariate weighted Cox regression with the factors whose P-values were less than 0.2, including the PTR at the weighed univariate Cox regression, the PTR showed a significantly decreased risk of cancer-related death (HR, $0.61 ; 95 \% \mathrm{CI}$, 0.40-0.94) (Table 3, Fig. 2).

\section{DISCUSSION}

In this study, we showed that asymptomatic CRC patients with unresectable metastases could achieve a survival gain by upfront PTR independently from the patients' likelihood of undergoing PTR based on their baseline characteristics by analysis with the IPTW method.

There are significant conflicts in the study of the impact of PTR on survival [6]. According to a study, PTR is performed in over $50 \%$ of patients with stage IV CRC, and many doctors still prefer routine use of this treatment. This may be because many current studies suggest that PTR can provide significant survival benefits $[7,10,12,15,23-25]$. In a Canadian study of stage IV CRC patients who underwent chemotherapy between and 2006 to 2010, 199 patients underwent upfront PTR, and 127 patients did not [24]. Ninety-one percent and $67 \%$ of patients received doublet chemotherapy and biologic agents, respectively. The median OS was significantly higher in the PTR group (27 months vs. 14 months). However, their results were not free from selection bias because there was a significant imbalance in the distribution of confounders, a higher rate of multiple organ metastasis (M1b), and worse performance status in the non-PTR group. In a post hoc analysis of 294 patients with non-resectable CRC metastases who were enrolled in a phase III randomized controlled trial (RCT), patients who underwent PTR before enrollment showed higher rates of OS than non-PTR patients [23]. However, the majority of PTR patients had single organ metastases (M1a) in the liver. Similarly, in a meta-analysis using survival data from 21 retrospective studies with a total of 44,226 patients with metastatic CRC, the mortality rate was lower with PTR than with chemotherapy alone. However, all but 2 of the studies in the analysis were single-center and the other 2 studies used administrative da-

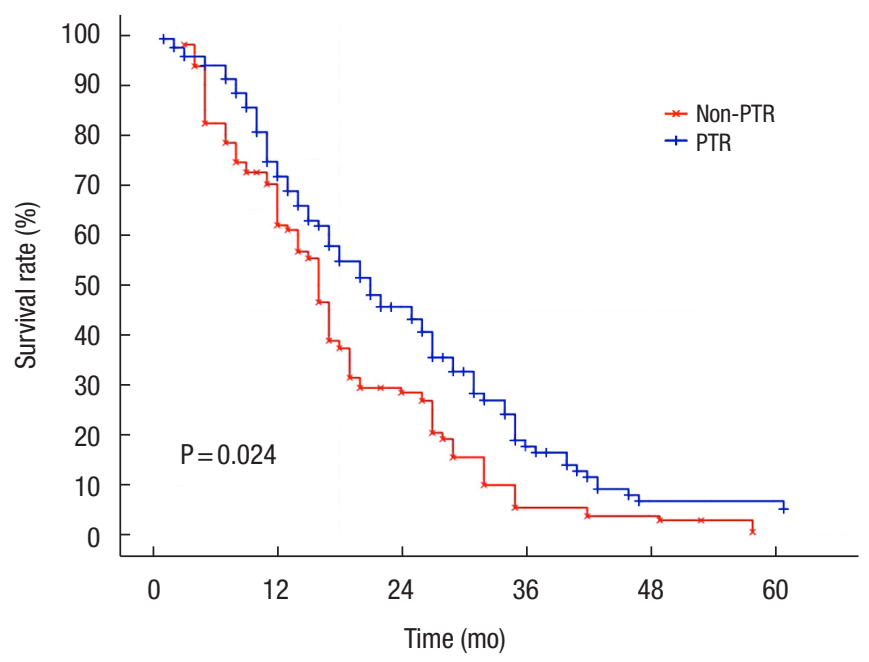

Fig. 2. Estimated survival of primary tumor resection (PTR) and non-PTR group by weighted multivariate Cox regression.

tabases with limited information about how patients were selected for the PTR [7].

These continued conflicts are because of the lack of firm results of the level I study. To answer this question, some clinical trials are ongoing [18, 19, 26, 27]. Kanemitsu et al. [28] reported their interim analysis results of an RCT at the 2020 American Society of Clinical Oncology meeting after early termination due to $50 \%$ of the expected event. They enrolled 160 patients, which was about half of the target enrollment number $(\mathrm{n}=280)$. The upfront PTR showed no survival benefit over the chemotherapy alone; the PTR group's median progression-free survival was 10.4 months (8.613.4 months) and that of chemotherapy alone was 12.1 months (9.4-13.2 months; HR, 1.08; 95\% CI, 0.77-1.50). Although the overall results of the remaining RCTs are expected, Kanemitsu's assertions imply that they would not be easy to see. Because all RCTs on this issue carry numerous intrinsic difficulties caused by strict eligibility criteria, patient preferences, and clinician bias lead to poor acceptance of the randomized assignment and difficulties in patient accrual.

In this retrospective study, we could see the survival benefit of upfront PTR. However, as mentioned earlier, this study has a fundamental limitation that cannot be free in this kind of study. The metastatic tumor burden and personal response to chemotherapy may be different for each patient. However, because of the lack of information on retrospective medical record review, we could not obtain detailed data. Although a sincere effort to reduce the selection bias, the patients selected from our database are those who visited our department of surgical oncology. Therefore, doctors in this department might have a strong tendency to prefer PTR. Additionally, because of the long period of study, most of the earlier decisions were not made with multidisciplinary team discussions. Inevitably, the patients enrolled in this study have a strong likeli- 
hood of getting upfront PTR. To overcome this issue, we used the weight from the average treatment effect on the treated (ATT). ATT is the one of the generally used treatment effects typically considered in causal inference. The other is the average treatment effect (ATE), which is the average effect of the treatment across the entire eligible sample, including the control sample's treatment effect. In a well-constructed RCT with perfect compliance to treatment assignment, both values must be the same. However, in the nonrandomized, observational studies like ours, the assignment of treatment can be influenced by various confounders such as patients' willingness or surgeon's preference for the PTR, which is not easily measurable. Endogenous selection bias is inevitable when using the ATE, referring to the entire study population, including those who did not receive the PTR. However, by using the ATT weight focusing only among those who received the PTR, we can estimate the effect of PTR in patients who received the real PTR by comparing in an assumed situation where the PTR patients might not receive the PTR [21, 29].

In conclusion, after minimizing the fundamental limitations of this retrospective study, this study demonstrated the beneficial impact of PTR on unresectable stage IV CRC patients. However, the debate about this issue will continue until the confirmative results of ongoing RCTs.

\section{CONFLICT OF INTEREST}

No potential conflict of interest relevant to this article was reported.

\section{ACKNOWLEDGMENTS}

This study was supported by a grant from the Korea Institute of Radiological and Medical Sciences (KIRAMS), funded by the Ministry of Science and ICT (MSIT), Republic of Korea (No. 50542-2020).

\section{REFERENCES}

1. Bray F, Ferlay J, Soerjomataram I, Siegel RL, Torre LA, Jemal A. Global cancer statistics 2018: GLOBOCAN estimates of incidence and mortality worldwide for 36 cancers in 185 countries. CA Cancer J Clin 2018;68:394-424.

2. Jung KW, Won YJ, Kong HJ, Lee ES. Cancer statistics in Korea: incidence, mortality, survival, and prevalence in 2016. Cancer Res Treat 2019;51:417-30.

3. Hur H, Oh CM, Won YJ, Oh JH, Kim NK. Characteristics and survival of Korean patients with colorectal cancer based on data from the Korea Central Cancer Registry data. Ann Coloproctol 2018;34:212-21.

4. Cirocchi R, Trastulli S, Abraha I, Vettoretto N, Boselli C, Montedori A, et al. Non-resection versus resection for an asymptomatic primary tumour in patients with unresectable stage IV colorectal cancer. Cochrane Database Syst Rev 2012;(8):CD008997.

5. National Comprehensive Cancer Network (NCCN). Clinical practice guidelines in oncology (NCCN guidelines): colon cancer [Internet]. Plymouth Meeting (PA): NCCN; 2020 [cited 2020 June 21]; Available from: https://www.nccn.org/professionals/physician_gls/ pdf/colon.pdf.

6. Hu CY, Bailey CE, You YN, Skibber JM, Rodriguez-Bigas MA, Feig BW, et al. Time trend analysis of primary tumor resection for stage IV colorectal cancer: less surgery, improved survival. JAMA Surg 2015;150:245-51.

7. Clancy C, Burke JP, Barry M, Kalady MF, Calvin Coffey J. A meta-analysis to determine the effect of primary tumor resection for stage IV colorectal cancer with unresectable metastases on patient survival. Ann Surg Oncol 2014;21:3900-8.

8. Faron M, Pignon JP, Malka D, Bourredjem A, Douillard JY, Adenis $\mathrm{A}$, et al. Is primary tumour resection associated with survival improvement in patients with colorectal cancer and unresectable synchronous metastases?: a pooled analysis of individual data from four randomised trials. Eur J Cancer 2015;51:166-76.

9. Niitsu H, Hinoi T, Shimomura M, Egi H, Hattori M, Ishizaki Y, et al. Up-front systemic chemotherapy is a feasible option compared to primary tumor resection followed by chemotherapy for colorectal cancer with unresectable synchronous metastases. World J Surg Oncol 2015;13:162.

10. Ahmed S, Fields A, Pahwa P, Chandra-Kanthan S, Zaidi A, Le D, et al. Surgical resection of primary tumor in asymptomatic or minimally symptomatic patients with stage IV colorectal cancer: a Canadian province experience. Clin Colorectal Cancer 2015;14: e41-7.

11. Alawadi Z, Phatak UR, Hu CY, Bailey CE, You YN, Kao LS, et al. Comparative effectiveness of primary tumor resection in patients with stage IV colon cancer. Cancer 2017;123:1124-33.

12. Xu Z, Becerra AZ, Fleming FJ, Aquina CT, Dolan JG, Monson JR, et al. Treatments for stage IV colon cancer and overall survival. J Surg Res 2019;242:47-54.

13. Yun JA, Huh JW, Park YA, Cho YB, Yun SH, Kim HC, et al. The role of palliative resection for asymptomatic primary tumor in patients with unresectable stage IV colorectal cancer. Dis Colon Rectum 2014;57:1049-58.

14. Wilkinson KJ, Chua W, Ng W, Roohullah A. Management of asymptomatic primary tumours in stage IV colorectal cancer: review of outcomes. World J Gastrointest Oncol 2015;7:513-23.

15. Kim MS, Chung M, Ahn JB, Kim CW, Cho MS, Shin SJ, et al. Clinical significance of primary tumor resection in colorectal cancer patients with synchronous unresectable metastasis. J Surg Oncol 2014;110:214-21.

16. van de Velde CJ, Boelens PG, Borras JM, Coebergh JW, Cervantes A, Blomqvist L, et al. EURECCA colorectal: multidisciplinary management: European consensus conference colon \& rectum. Eur J Cancer 2014;50:1.

17. Van Cutsem E, Cervantes A, Adam R, Sobrero A, Van Krieken $\mathrm{JH}$, Aderka D, et al. ESMO consensus guidelines for the manage- 
ment of patients with metastatic colorectal cancer. Ann Oncol 2016;27:1386-422.

18. 't Lam-Boer J, Mol L, Verhoef C, de Haan AF, Yilmaz M, Punt CJ, et al. The CAIRO4 study: the role of surgery of the primary tumour with few or absent symptoms in patients with synchronous unresectable metastases of colorectal cancer: a randomized phase III study of the Dutch Colorectal Cancer Group (DCCG). BMC Cancer 2014;14:741.

19. Rahbari NN, Lordick F, Fink C, Bork U, Stange A, Jäger D, et al. Resection of the primary tumour versus no resection prior to systemic therapy in patients with colon cancer and synchronous unresectable metastases (UICC stage IV): SYNCHRONOUS: a randomised controlled multicentre trial (ISRCTN30964555). BMC Cancer 2012;12:142.

20. Feo L, Polcino M, Nash GM. Resection of the primary tumor in stage IV colorectal cancer: when is it necessary? Surg Clin North Am 2017;97:657-69.

21. Austin PC. An introduction to propensity score methods for reducing the effects of confounding in observational studies. Multivariate Behav Res 2011;46:399-424.

22. Austin PC. Balance diagnostics for comparing the distribution of baseline covariates between treatment groups in propensity-score matched samples. Stat Med 2009;28:3083-107.

23. Ferrand F, Malka D, Bourredjem A, Allonier C, Bouché O, Louafi $S$, et al. Impact of primary tumour resection on survival of patients with colorectal cancer and synchronous metastases treated by chemotherapy: results from the multicenter, randomised trial
Fédération Francophone de Cancérologie Digestive 9601. Eur J Cancer 2013;49:90-7.

24. Ahmed S, Leis A, Chandra-Kanthan S, Fields A, Reeder B, Iqbal N, et al. Surgical management of the primary tumor in stage IV colorectal cancer: a confirmatory retrospective cohort study. J Cancer 2016;7:837-45.

25. Xu J, Ma T, Ye Y, Pan Z, Lu D, Pan F, et al. Surgery on primary tumor shows survival benefit in selected stage IV colon cancer patients: a real-world study based on SEER database. J Cancer 2020;11:3567-79.

26. Moritani K, Kanemitsu Y, Shida D, Shitara K, Mizusawa J, Katayama $\mathrm{H}$, et al. A randomized controlled trial comparing primary tumour resection plus chemotherapy with chemotherapy alone in incurable stage IV colorectal cancer: JCOG1007 (iPACS study). Jpn J Clin Oncol 2020;50:89-93.

27. Kim CW, Baek JH, Choi GS, Yu CS, Kang SB, Park WC, et al. The role of primary tumor resection in colorectal cancer patients with asymptomatic, synchronous unresectable metastasis: study protocol for a randomized controlled trial. Trials 2016;17:34.

28. Kanemitsu Y, Shitara K, Mizusawa J, Hamaguchi T, Shida D, Komori K, et al. A randomized phase III trial comparing primary tumor resection plus chemotherapy with chemotherapy alone in incurable stage IV colorectal cancer: JCOG1007 study (iPACS). J Clin Oncol 2020;38(4 Suppl):7.

29. Garrido MM, Dowd B, Hebert PL, Maciejewski ML. Understanding treatment effect terminology in pain and symptom management research. J Pain Symptom Manage 2016;52:446-52. 